\title{
Remaining Useful Life Assessment of Lithium-ion Battery based on HKA-ELM Algorithm
}

\author{
Jing Yang ${ }^{1,2,3}$, Zhen Peng ${ }^{4}$, Zhaodi Pei ${ }^{1,2,3}$, YongGuan ${ }^{1,2,3}$, Huimei Yuan ${ }^{1,2,3}$, Lifeng Wu ${ }^{1,2,3^{*}}$ \\ ${ }^{1}$ College of Information Engineering, Capital Normal University, Beijing 100048, China \\ ${ }^{2}$ Beijing Key Laboratory of Electronic System Reliability Technology, Capital Normal University, \\ Beijing 100048, China \\ ${ }^{3}$ Beijing Advanced Innovation Center for Imaging Technology, Capital Normal University, Beijing \\ 100048, China \\ ${ }^{4}$ Information Management Department, Beijing Institute of Petrochemical Technology, Beijing 10217 , \\ China \\ *E-mail: wulifeng@cnu.edu.cn
}

doi: $10.20964 / 2018.10 .51$

Received: 5 May 2018/ Accepted: 30 July 2018 / Published: 1 September 2018

Accurately predicting the remaining useful life (RUL) of lithium-ion batteries is very important to battery management systems (BMS). Recently, Extreme Learning Machine (ELM) algorithm has been applied to RUL prediction of lithium-ion batteries. However, the input weights and biases of the ELM algorithm are generated randomly, which affect its prediction accuracy. In this paper, we use the heuristic Kalman algorithm (HKA) to optimize the input weights and biases of the ELM algorithm. The mean square error (MSE) obtained from the ELM is used as the cost function of the HKA algorithm, and the optimized particles in the HKA are used as the weights and biases of the ELM predictor. In this work, the HKA-ELM method is introduced firstly, then, we perform experiments on the battery data set to verify the proposed algorithm, and compare with other algorithms. Results show that our proposed method has better prediction accuracy than related works.

Keywords: Lithium-ion battery, RUL prediction, ELM, HKA

\section{$\underline{\text { FULL TEXT }}$}

(C) 2018 The Authors. Published by ESG (www.electrochemsci.org). This article is an open access article distributed under the terms and conditions of the Creative Commons Attribution license (http://creativecommons.org/licenses/by/4.0/). 